\title{
Clinical Study of Heparin-Free Hemodialysis with the Inside of Hollow Fibers in Dialyzer Coated by Human Albumins
}

\author{
Xixin $\mathrm{Wu}^{\mathrm{a}}$ Huaji Chen ${ }^{\mathrm{b}}$ \\ aDepartment of Nephrology, The Fifth Affiliated Hospital, Zhong Shan University, Zhuhai City, and \\ bDepartment of Nephrology, Guangdong Farm Center Hospital, Zhanjiang City, Republic of China
}

\section{Key Words}

Heparin-free $\cdot$ Hemodialysis $\cdot$ Albumins $\cdot$ Scanning

electron microscopy $\cdot$ Renal failure

\begin{abstract}
Background/Objective: To investigate the therapeutics of heparin-free hemodialysis (HD) with the inside of hollow fibers in a dialyzer coated with human albumins (HFHd-A1). Methods: Clinical contrast study between HFHd-Al and heparin-free HD with hollow fibers in dialyzer by normal saline flush (HFHd-NS) was performed by adopting the cross-over design method. The dialysis effect, the effect on life signs, blood platelet functions, change of dialyzer fiber-bundle volume, appearance image, and image of scanning electron microscopy of the inside of hollow fibers in dialyzer after dialysis and the biocompatibility of dialyzer of two HD methods were compared. Results: HFHd-Al could overcome all shortcomings of HFHd-NS and had more merits and increased expenses for HFHd-Al were not high. Conclusion: HFHdAl was an effective and feasible method which could salvage patients with bleeding diseases or bleeding tendency and who need HD.
\end{abstract}

There will be a serious consequence of accelerating the bleeding in the body if hemodialysis (HD) is performed using heparin as an anticoagulant in patients with renal failure with bleeding [1]. At present, it is thought that heparin-free HD (HFHd) with hollow fibers in a dialyzer with normal saline (NS) flush (HFHd-NS) is an effective and feasible method which could treat these patients [2, 3]. But there are still shortcomings in the HFHd-NS method: (1) An excess of NS as infused into the patient during the HFHd-NS period, so that the fluctuation of cardiac anterior load in the patient was increased, which was not advantageous for HD in patients with cardiac insufficiency or fluid overload. (2) When HD is performed by means of this method in some bleeders, coagulation might occur in the dialyzer, so that HD would fail. To overcome those shortcomings and further perfect HFHd, we designed the HFHd method of using albumins (A1) to coat the dialyzer before HD.

\section{Material and Methods}

\section{Patient Selection and Grouping}

There were 19 uremic patients in our study, 9 male 10 female, age 29-67 years, 8 cases with bleeding and 11 cases with bleeding tendency. All patients had no DIC and none had recently used any anticoagulant. According to a cross-over design method, HFHds were per-

\begin{tabular}{ll}
\hline KARGER & ( 2002 S. Karger AG, Basel \\
0028-2766/02/0924-0925\$18.50/0 \\
$\begin{array}{l}\text { Fax +41613061234 } \\
\text { E-Mail karger@karger.ch } \\
\text { www.karger.com }\end{array}$ & $\begin{array}{l}\text { Accessible online at: } \\
\text { www.karger.com/journals/nef }\end{array}$
\end{tabular}

Wu Xixin

8th building Apt. $6 \mathrm{~A}$

Hong Shan An Ju Yuan, People west road

Zhuhai City, 519000 Guangdong Province (People's Republic of China)

E-Mail zhwxxzx@pub.zhuhai.gd.cn/cuw@21cn.com 
Table 1. Comparison between dehydration quantity and NS quantity flushed into the body and ultrafiltrated quantity (in liters) of the two groups

\begin{tabular}{llll}
\hline & $\begin{array}{l}\text { Dehydration } \\
\text { quantity }\end{array}$ & $\begin{array}{l}\text { NS quantity } \\
\text { flushed into body }\end{array}$ & $\begin{array}{l}\text { Ultrafiltrated } \\
\text { quantity }\end{array}$ \\
\hline Group HFHd-A1 $(\mathrm{n}=30)$ & $2.303 \pm 1.288$ & $0.428 \pm 0.195^{*}$ & $\begin{array}{l}2.812 \pm 1.496 \# \\
\text { Group HFHd-NS }(\mathrm{n}=30)\end{array}$ \\
\hline
\end{tabular}

Values expressed as mean $\pm \mathrm{SD}$. Only significant results are presented. Compared with group HFHd-NS: * $\mathrm{p}<0.005$, \# $\mathrm{p}<0.05$.

HFHd-A1 = Heparin-free hemodialysis with the inside of hollow fibers in dialyzer coated with human albumins; HFHd-NS = heparin-free HD with hollow fibers in dialyzer by normal saline flush. $\mathrm{NS}=$ Normal saline. formed 2-6 times early or late and alternatively in each patient according to the same time interval after umpteen times of HFHdNS: In one group, the inside of the hollow fibers in the dialyzer was coated with A1 before HD and we would flush the dialyzer with NS every 90 min during HD (group HFHd-A1); another group did not use A1 to coat the dialyzer before HD and according to the HFHdNS frequent-use method, the dialyzer was flushed with NS every 20 min during HD (group HFHd-NS). There were 30 HDs in each group.

\section{HD Conditions and Method}

HD machine, dialysis catheter, dialyzer type and reused method of dialyzer were entirely identical between the two groups. The dialysate ingredient, flow rate, temperature, electric conductivity, blood flow rate and duration of dialysis were basically identical between the two groups. HFHd-NS method: The dialysis catheter was flushed by 2 liters of NS and then discarded before HD. During HD, $200 \mathrm{ml} \mathrm{NS}$ were put into the arterial pipeline of the blood pathway by the blood pump and at the same time the pipeline in front of the blood pump was nipped for interdicting blood flow. The bestraddling membrane pressure in the dialyzer was augmented for blood remains and the increasing NS ultrafiltrated out. HFHd-A1 method: The inside of hollow fibers in the dialyzer were coated with $1 \% \mathrm{~A} 1$ before HD. During HD the dialyzer was flushed with $200 \mathrm{ml}$ NS every $90 \mathrm{~min}$ and then NS into the body as discarded by augmenting the bestraddling membrane press in the dialyzer.

\section{Observational Target Mensuration}

Mean arterial pressure (MAP), heart rate (HR), white blood cell (WBC) and $\mathrm{C}_{3}$ of the two groups were mensurated before $\mathrm{HD}$ and, at 15,120 and 240 min during HD. The serum creatinine (SCr) and blood urea nitrogen (BUN) of the two groups were mensurated by collecting arterial blood before and after HD for calculating the descent rate of $\mathrm{SCr}$ and $\mathrm{BUN}$. The 1-min, 5-min, most platelet aggregation (PagT1', 5' and $\mathrm{M})$ and platelet adhesive tests (PadT) of the two groups for each HD were mensurated by collecting arterial blood before and at $120 \mathrm{~min}$ during HD. The dialyzer fiber-bundle volumes (FBV) of the two groups for each HD were mensurated before and after HD. The one-time HD in every group not flushed with NS within $60 \mathrm{~min}$ of $\mathrm{HD}$, as for new dialyzers used in this HD by the two groups were managed strictly according to the coequal method. After the dialyzer was used, images were taken of the 20 hollow fiber bundles (about $1 \mathrm{~cm}$ in length) at the center at middle segment of the arterial end of the dialyzer. After fixation by $95 \%$ alcohol, 5 of the 20 hollow fiber bundles were taken out randomly and were dissected under a magnifying microscope and observed. Some images of the inside of hollow fiber bundles were taken by the H-3010 scanning electron microscopy after being dehydrated and sprayed gold. The method was that one image at the same position of every end of the inside of every hollow fiber bundle of the two groups was taken every time and 10 images could be taken per group. The RBC counts for every image were calculated. All the images from each group were observed and contrasted in parallel.

\section{Statistics}

Values were expressed as mean \pm SD. Comparison between numerical variables was performed according to Student's t test (twotailed, paired).

Differences were considered significant if the probability of error was less than $5 \%$, or according to $95 \%$ confidence intervals.

The SPSS8.0 software (SPSS, Inc, Chicago, Ill., USA) was used for data management.

\section{Results}

(1) Comparison of dialyser effect: For comparison of the dehydrate quantity, the NS quantity flushed into the body, and the ultrafiltrated quantity of the two groups see table 1. The BUN and SCr values of the two groups after HD dropped significantly in comparison with the levels before HD ( $p<0.002)$. There was no significant difference for the drop rate of these targets between the two groups ( $p>0.1$ or 0.2$)$. There was no significant difference between the two groups for the clean rate of the dialyzer to $\mathrm{SCr}$ from when HD was performed to $60 \mathrm{~min}(\mathrm{p}>0.5)$.

(2) Effect on life signs: There was no significant change in MAP or HR in the course of each HD session between the two groups $(\mathrm{p}>0.05)$.

(3) For the effect on platelet function, see table 2.

(4) FBV changes in the two groups: There were no significant differences for FBV before HD between the two 
Table 2. HFHd-A1 and HFHd-NS effect on platelet function

\begin{tabular}{llllll}
\hline $\begin{array}{l}\text { Observational } \\
\text { target }\end{array}$ & \multicolumn{2}{l}{ Group HFHd-A1 $(\mathrm{n}=30)$} & & \multicolumn{2}{l}{ Group HFHd-NS $(\mathrm{n}=30)$} \\
\cline { 2 - 3 } \cline { 5 - 6 } & before HD & 120 min into HD & & before HD & 120 min into HD \\
\hline PAgT1' & $13.6 \pm 4.2$ & $13.3 \pm 5.2^{*}$ & & $16.4 \pm 12.1$ & $23.3 \pm 11.7$ \\
PAgT5' & $21.8 \pm 9.1$ & $21.6 \pm 7.9^{*}$ & & $27.6 \pm 16.5$ & $33.8 \pm 15.6$ \\
PAgTM & $29.7 \pm 11.2$ & $29.6 \pm 11.6^{*}$ & & $30.5 \pm 15.7$ & $41.6 \pm 13.9$ \\
PAdT & $25.1 \pm 5.3$ & $26.0 \pm 3.9^{*}$ & & $27.6 \pm 5.3$ & $30.1 \pm 6.1$ \\
\hline
\end{tabular}

Values expressed as mean $\pm \mathrm{SD}$. Only significant results are presented. $*$ Compared with HFHd-NS group when 120 min into HD, $\mathrm{p}<0.05$.

HFHd-A1 = Heparin-free hemodialysis with the inside of hollow fibers in dialyzer coated with human albumins; HFHd-NS = heparin-free HD with hollow fibers in dialyzer by normal saline flush; $\mathrm{PAgT1}^{\prime}=1 \mathrm{~min}$ platelet aggregation test; $\mathrm{PAgT}^{\prime}=5$ min platelet aggregation test PAgTM = most platelet aggregation test PAdT = platelet adhesive test $\mathrm{HD}=$ hemodialysis. groups $(\mathrm{p}>0.2)$. After HD, FBV of the HFHd-A1 group was significantly higher than in the HFHd-NS group ( $\mathrm{p}<$ 0.005). FBV of the HFHd-A1 group after HD accounts for $95.1 \%$ before HD and that of the HFHd-NS group $86.7 \%$.

(5) Comparison of the appearance imaging and scanning electron microscopy imaging of the inside of the hollow fibers between the two groups after HD: (a) Appearance imaging: The arterial end of the dialyzer in the HFHd method was slightly discolored red and the arterial end of the dialyzer in the HFHd-A1 method was clean. (b) Comparison of the RBC count (values expressed as mean $\pm \mathrm{SD}$ ) on the scanning electron microscopy between the two groups: The RBC count $(29 \pm 26, \mathrm{n}=10)$ in the HFHd group was significantly higher than in the HFHd-A1 group $(4 \pm 3, \mathrm{n}=10)(\mathrm{p}<0.01)$. (c) Comparison of the scanning electron microscopy between the two groups: The RBC on the inside of the hollow fibers in the dialyzer in the HFHd method were of a much greater amount and were held together by a great deal of fibrin which conglutinated on the wall at the end of the hollow fibers, showing that a blood coagulating process had occurred in the dialyzer during HD. Only a few RBC attached to the hollow fiber wall were observed on the scanning electron microscopy image in the HFHd-A1 method but there was the fibrin.

(6) Between groups comparisons of the biocompatibility of dialyzers: The frequency of tight-chestedness, headache and vomiting in the course of HD in the HFHd-A1 group was lower than in the HFHd-NS group. The degree of drop of the $\mathrm{C}_{3}$ and WBC of the HFHd-A1 group at 15 min into HD was significantly less than in the HFHd-
NS group $(p<0.01$ or 0.05$)$. At 120 min into HD, the degree of drop of the $\mathrm{C}_{3}$ of the HFHd-A1 group was still significantly less than that of the HFHd-NS group $(\mathrm{p}<$ $0.05)$.

\section{Discussion}

(1) The problem about the water balance in HD: Table 1 shows that the NS quantity which was flushed into the body and the ultrafiltrated quantity in the method HFHd-A1 were significantly less than those in the HFHd-NS method. In the former, MAP descended steadily and there was little change in HR, and in the latter, they fluctuated significantly. This prompted HR quickening adjustability because the NS quantity which was flushed into the body was more and was not ultrafiltrated out in a short time. Selecting HFHd-A1 was safer than HFHd-NS for the patients with bleeding and cardiac insufficiency or fluid overload.

(2) The A1 membrane did not affect the solute exchange: The A1 membrane which coated the dialyzer in the HFHd-A1 method did not affect solute exchange, and the ability to purge to the toxicity substance of the dialyzer which was coated by A1 was the same as in the method HFHd-NS.

(3) The A1 membrane could improve the biocompatibility of the dialyzer. Gao et al. [4] reported a frequent WBC decrease, and alexin activation and consumption in HD patients could debase the ability to resist infection. Our result 6 attests that the A1 membrane which coated the dialyzer could improve the biocompatibility. There- 
by, reducing the patient's discomfort during HD and toning up the ability to resist infection.

(4) The coagulation extent in the dialyzer during the period of HFHd-A1 was lighter than HFHd-NS: It has been reported that the coagulation extent in the dialyzer could be determined according to the remains found in the dialyzer after treatment $[2,5]$. Result 4 shows that the coagulation extent in the dialyzer during the period of HFHd-A1 was less than with HFHd-NS.

(5) The A1 membrane effect on the coagulation course in the dialyzer: We have observed the appearance image and the scanning electron microscopic scanning of the inside of hollow fibers and the RBC count in two new dialyzers. The results prove that the A1 membrane could restrain RBC conglutination and prevent the coagulation course. But the mechanism is still not clear.

(6) The A1 membrane could prevent from conglutination and collective platelet functions: Result 3 prompted that the A1 membrane could prevent conglutination and collective platelet functions.

Therefore, the three aspects could be improved if HFHd-A1 therapy was selected in the field of treatment for uremic patients with bleeding or bleeding tendency, in comparison with HFHd-NS: (1) The coagulation extent in the dialyzer was alleviated during the period of HFHd. (2) Normal saline flushes were reduced. Thus, the fluctuation of cardiac anterior load in the patients was alleviated during the period of HD, which would be safer if HD was performed in uremic patients with cardiac insufficiency or fluid overload. (3) The biocompatibility of the dialyzer and pipeline had been improved and thereby the discomfort of patients during HD was reduced and the ability to resist infection was raised.

Cost increases for the HFHd-A1 method was not great and the $20 \%$ human albumins which were needed per HD session amounted to only $12.5 \mathrm{ml}$.

\section{References}

1 Wang Xiaoyun: The bleeding complication and anticoagulation treatment for 8016 time HDs. Jiangsu Medicine 1989;1:9.

2 Ludlow MK: Heparin-free dialysis. ANNA J 1980;16:295.

3 Geary DF: Low-dose and heparin-free hemodialysis in children. Pediatr Nephrol 1991;5:220.
4 Gao Xiulin, Liu Huilian: The comparison of the biocompatibility of Acetate fibers membrane dialyzers and Cuprophan membrane dialyzers. Chin J Dial Artif Organs 1991;2:8-11.

5 Liao Liudan: Clinical report of repetitive use of hollow fiber dialyzers. Chin J Organs Transplant 1985;6:14-16. 